\title{
The Investigation of Tramadol Dependence with No History of Substance Abuse: A Cross-Sectional Survey of Spontaneously Reported Cases in Guangzhou City, China
}

\author{
Haoran Zhang ${ }^{1,2}$ and Zhimin Liu ${ }^{2}$ \\ ${ }^{1}$ School of Public Health, Peking University, Beijing 100191, China \\ ${ }^{2}$ National Institute on Drug Dependence, Peking University, 38 Xueyuan Road Haidian District, Beijing 100191, China \\ Correspondence should be addressed to Zhimin Liu; zhiminliu@bjmu.edu.cn
}

Received 10 April 2013; Revised 14 July 2013; Accepted 14 August 2013

Academic Editor: Eiichi Kumamoto

Copyright ( $\odot 2013 \mathrm{H}$. Zhang and Z. Liu. This is an open access article distributed under the Creative Commons Attribution License, which permits unrestricted use, distribution, and reproduction in any medium, provided the original work is properly cited.

The study was to survey and assess the drug dependence and abuse potential of tramadol with no history of substance abuse. Subjects of tramadol dependence with no prior history of substance abuse were surveyed by interview. Physical dependence of tramadol was assessed using 10 items opiate withdrawal scale (OWS), and psychological dependence was assessed by Addiction Research Center Inventory-Chinese Version (ARCI-CV). Twenty-three male subjects (the median age was $23.4 \pm 4.1$ years) referred to the addiction unit in Medical Hospital of Guangzhou with tramadol abuse problems were included in this cross-sectional study. The control group included 87 heroin addicts, 60 methamphetamine (MA) abusers, and 50 healthy men. The scores of OWS of tramadol were 0.83-2.30; the mean scores of identifying euphoric effects-MBG, sedative effects-PCAG, and psychotomimetic effects-LSD of ARCI were $8.96 \pm 3.08,6.52 \pm 3.25$, and $6.65 \pm 2.50$, respectively, $F=4.927, P<0.001$. Scores of MBG scale in tramadol did not differ from those in heroin and MA groups $(P>0.05)$ but were higher than those in healthy men $(P<0.05)$. Tramadol with no history of substance abuse has a clear risk of producing high abuse potential under the long-term infrequent abuse and the high doses.

\section{Introduction}

Tramadol, marketed in Germany by Grunenthal since 1977, is a centrally acting analgesic with weak $\mu$-opioid agonist properties and inhibition of norepinephrine (NA) and serotonin reuptake. The drug is most widely used in moderate to severe acute and chronic pain. It has been postulated that tramadol achieves its analgesic activity from an M1 metabolite with potent opioid properties and through inhibition of reuptake of monoamines [1]. Prior to its United States (US) approval in 1995, tramadol was marketed in Europe for approximately 20 years with little evidence of abuse. The abuse probability of tramadol in USA was $2-3 / 100,000$ and then declined to 1/100,000 [2]. World Health Organization (WHO) attached great importance to the abuse and dependence of tramadol, and four estimates were done. From 1992 to 2006, tramadol dependence was evaluated for four times by Expert Committee on Drug Dependence (ECDD) [3-6]. Because the available information was still not enough, it could not decide to control tramadol internationally. Although tramadol abuse is a low-level worldwide, the nonmedical purpose abuse of tramadol was popular in China since its first marketing in China in the early 1990s as a noncontrolled analgesic, especially in opiate addicts and adolescents. In order to prevent the abuse of tramadol and manage the clinical application of tramadol, in 2007, the State Food and Drug Administration of China issued the newest version of measures for psychotropic drugs administration and tramadol was controlled as the second category of psychotropic drugs [7]. After that, the incidence of tramadol abuse has declined to a low level. According to the report of National Drug Abuse Monitoring, the proportion of tramadol use among drug abusers increased from $0.2 \%$ in 2004 to $16.0 \%$ in 2006 ; the trend of tramadol use varied very smoothly from 2007 to 2009; however, the proportion of tramadol use among drug abusers declined sharply from $13.3 \%$ in 2009 to $3.4 \%$ 
in 2011 [8-11]. However, it is worth noting that tramadol abuse was still prevalent in some regions, and tramadol abuse shows a property of regional distribution. Among those, the percentage of tramadol use was highest in South China (5.1\%), and tramadol popular use in Guangdong province was still relatively high [11]. According to statistics of Drug Monitoring Center of Guangdong Province, the number of tramadol abusers increases from 11 persons in 2004 to 4,492 persons in 2006 in Guangdong province [12]. Based on these reasons, all tramadol patients were recruited in Guangzhou city, Guangdong Province.

Tramadol is generally considered to be safe and thought to have minimal potential for abuse. Despite a range of studies, still no consensus data exist on the dependence potential of tramadol. Studies from animal experiment hold controversial finding, one is that tramadol was not likely to induce tolerance and physical dependence in mice [13] and the other is that conditioned place preference (CPP) in rats was produced $[14,15]$. Though tolerance and dependence were not described after repeated administration of tramadol in human $[16,17]$, case reports that tramadol can lead to dependence continue to emerge $[18,19]$. It was suggested that the abuse liability of tramadol may be greater than hitherto assumed [20].

Hence, data for the dependence and abuse potential of tramadol are conflicting, and important information is still missing regarding cases of tramadol dependence and abuse in China. The objective of this study was to evaluate drug dependence and abuse potential of tramadol, using tramadol abusers spontaneously referred to the addiction unit of Medical Hospital in Guangzhou city between July 2012 and December 2012.

\section{Methods}

2.1. Ethical Statement. The study was approved by the ethical committee of Peking University Health Center (Grant number IRB00001052-10026). Before the interviews were conducted, subjects were informed about the purpose of this study and confidentiality of all information they would provide. Respondents' consent was gathered. The survey was conducted in an isolated room, and the survey procedures were designed to protect privacy of subjects by allowing for anonymous and voluntary participation. Participants in the study could stop at any time if they desired.

2.2. Subjects. Identification and collection of relevant cases were conducted from 1 July 2012 to 1 January 2013. All available information in the included tramadol cases was registered: age, sex, occupation, marital status, educational status, concomitant medication, prescribed and ingested doses of tramadol, duration of tramadol abuse, and other important information. Subjects reported to have a history of continuous tramadol abuse for 7 days in a week or those who had life-time used tramadol for 6 months at least were further reviewed by the American Psychiatric Association Diagnostic and Statistical Manual of Mental Disorders, fourth revision (DSM-IV). Only subjects who fulfilled these criteria were finally included in the final analysis. Though the identification of tramadol dependence in this study was conducted based on DSM-IV criteria, the diagnosis in the detoxification unit has no such information. Generally, patients in the drug dependence agencies may require tramadol detoxification or dose reduction.

The inclusion criteria were (1) male; (2) 18 years old or above; (3) the primary abused drug of choice was tramadol; (4) willing to sign an informed consent; and (5) adhered sufficiently to the study protocol. Meanwhile, the exclusionary criteria were made: (1) unwilling to participate or could not provide written informed consent; (2) could not discriminate the primary drug of abuse; (3) severe psychotic disorders; (4) severe body diseases (e.g., prostrate disease of liver or kidney); (5) be apt to drop out; and (6) deficiency in or lack of language and comprehensive problems. The criteria for controls were (1) men aged 18 and above; (2) for healthy controls, without a history of drug abuse before the study and no evidence of drug abuse during study; (3) for heroin addicts and methamphetamine abusers, predominate drugs of choice were heroin and methamphetamine in the baseline survey, respectively; (4) provided informed consent. Overall, a total of 23 eligible tramadol addicts, 85 heroin addicts, 60 methamphetamine addicts, 45 smokers, and 50 healthy controls were recruited.

2.3. Data Collection and Measures. The study was a crosssectional survey. Before the formal survey, a pilot trial was administrated. Researchers participated in the investigation were trained by empirical experts on specific aspects of survey techniques. Each sample was briefly informed about the purposes of the investigation, in order to seek their support and cooperation. After that, a written informed consent was obtained from all participants. A questionnaire with detailed baseline variables was offered by National Institute on Drug Dependence, Peking University. Current sociodemographic characteristics, history of substance use, and information of ARCI were obtained from a face-to-face interview. All finished questionnaires were reviewed by researchers for completeness and consistency.

Physical dependence of tramadol was assessed using 10 items opiate withdrawal scale (OWS), which composed of 10 symptoms/signs. A 4-point scale was used to rate each symptoms/signs: zero (0), mild (1), moderate (2), and severe (3). Subjects were asked to rate their symptoms according to severity of previous experienced tramadol withdrawal. Psychological dependence was assessed by association test of Addiction Research Center Inventory-Chinese Version (ARCI-CV) [21]. The 40-item ARCI is a true-false questionnaire with three empirically derived scales that are sensitive to the effects of a variety of classes of abused drugs. The derived scales were the MBG (morphine-benzedrine group), a measure of drug-induced euphoria; the PCAG (pentobarbitalchlorpromazine group) scale, which measured sedation; and the LSD (lysergic diethylamide) scale, which measures dysphoria and somatic symptoms. The same method which priority evaluated drug dependence of dihydroetorphine and buprenorphine was used in this study. 
TABLE 1: Characteristics of demographic and history of drug use.

\begin{tabular}{|c|c|c|c|c|c|c|}
\hline Variables & $\begin{array}{c}\text { Tramadol } \\
(n=23) \\
n(\%)\end{array}$ & $\begin{array}{c}\text { Heroin } \\
(n=85) \\
n(\%)\end{array}$ & $\begin{array}{c}\mathrm{MA}^{\#} \\
(n=60) \\
n(\%)\end{array}$ & $\begin{array}{c}\text { Healthy men } \\
(n=50) \\
n(\%)\end{array}$ & $F / \chi^{2}$ & $P$ \\
\hline \multicolumn{7}{|l|}{ Age (years) } \\
\hline $\bar{x} \pm s$ & $23.4 \pm 4.1$ & $25.7 \pm 9.16$ & $23.6 \pm 8.1$ & $22.9 \pm 3.1$ & 1.148 & 0.267 \\
\hline Range & $(18-34)$ & $(18-61)$ & $(20-56)$ & $(19-29)$ & & \\
\hline \multicolumn{7}{|l|}{ Education } \\
\hline Junior high school & $9(39.1)$ & $28(32.9)$ & $19(31.7)$ & $16(32.0)$ & 1.941 & 0.115 \\
\hline Senior high school and above & $14(60.9)$ & $57(67.1)$ & $41(68.3)$ & $34(68.0)$ & & \\
\hline \multicolumn{7}{|l|}{ Marital status } \\
\hline Single & $15(65.2)$ & $56(65.9)$ & $31(51.7)$ & $43(86.0)$ & 1.252 & 0.207 \\
\hline Others* & $8(34.8)$ & $29(34.1)$ & $29(48.3)$ & $7(14.0)$ & & \\
\hline \multicolumn{7}{|l|}{ Ethnicity } \\
\hline Han ethnicity & $21(91.3)$ & $70(82.4)$ & $59(98.3)$ & $49(98.0)$ & 1.094 & 0.236 \\
\hline Others & $2(8.7)$ & $15(17.6)$ & $1(1.7)$ & $1(2.0)$ & & \\
\hline \multicolumn{7}{|l|}{ Occupation } \\
\hline Unemployed ${ }^{\mathbf{\nabla}}$ & $7(30.4)$ & $31(36.5)$ & $19(31.7)$ & $30(60.0)$ & 0.289 & $0.591^{\#}$ \\
\hline Employed & $16(69.6)$ & $54(63.5)$ & $41(68.3)$ & $20(40.0)$ & & \\
\hline
\end{tabular}

Including students.

${ }^{*}$ Including married, divorced, and others.

"Methamphetamine.

2.4. Statistical Analysis. Statistical analysis was completed using the SPSS computer software for Microsoft Windows (SPSS Inc, Chicago, IL, USA). Quantitative symmetrically distributed variables were described by using the mean and standard deviation, whereas quantitative variables with a skewed distribution were described by using the median. Nonparametric statistical tests were undertaken when the distribution was not normal. Descriptive statistics were used to examine distributions of demographic and history of drug abuse. Differences among subgroups of ARCI were explored using $t$-test or one-way analysis of variance (ANOVA). All tests of statistical significance were two-tailed and used an alpha level of 0.05 .

\section{Results}

3.1. Characteristics of Demographic and History of Drug Use. Demographic characteristics and history of drug use were summarized in Tables 1 and 2. At baseline, no significant difference was observed in respondent's average age, educational status, marital status, ethics, and occupation $(P>0.05)$ across groups.

Of the 23 tramadol patients, the median age was (23.4 \pm 4.1) years and ranged between 18 and 34 years and $73.9 \%$ $(17 / 23)$ of cases were lower than 25 years. $87.0 \%(20 / 23)$ of the sample had no previous history of drug abuse before tramadol abuse, and $90.0 \%$ (21/23) were on tramadol alone or $10.0 \%$ on poly drugs. The drugs most frequently used in combination with tramadol were cough syrup (5 subjects) and methamphetamine (MA) (3 subjects). The median dose was $750 \mathrm{mg} /$ per time at frequency and $2000 \mathrm{mg} /$ per time at most. The median duration of tramadol use was $61.8 \pm$
30.2 months (ranged from 5 months up to 112 months). The median age of first tramadol use was $18.3 \pm 4.0$ years. All 23 tramadol users reported that the route of administration was oral method. Instigating by friends or other acquaintances was the most reason of tramadol use for 23 cases, which accounts for $60.9 \%$ (14/23), and there were $39.1 \%$ reported that the reason of tramadol use was curiosity or simulating. As for the source of tramadol, the top three were private clinic or pharmacy (12/23), normal pharmacy (6/23), and black market (6/23). $69.6 \%(16 / 23)$ of subjects have at least one time or above admission to detoxification or drug reduction.

3.2. Measure of Physical Dependence. As mentioned in the methods, physical dependence was rated by scores of 10 items opiate withdrawal scale (OWS). In total, the range of mean scores of OWS scale was $0.83-2.30$. Of those, $95.7 \%$ (22/23) of the patients had at least one seizure. Ratings on the insomnia $(2.30 \pm 0.93)$, yawning $(1.83 \pm 0.98)$, and runny eyes ( $1.61 \pm$ 1.23) were the top three increased by tramadol. However, the differences of ratings of each sign/symptom between tramadol and heroin did not reach statistical significance $(P>0.05)$.

3.3. Measure of Psychological Dependence. Table 3 details the measure of psychological dependence of tramadol and comparators. The mean scores of three scales in identifying euphoric effects-MBG, sedative effects-PCAG, and psychotomimetic effects-LSD of ARCI were $8.96 \pm 3.08$, $6.52 \pm 3.25$, and $6.65 \pm 2.50$, respectively. One-way analysis of variance indicated that the variation in 3 scales was significant $(F=4.927, P<0.001)$. On the all three scales of ARCI 
TABLE 2: History of drug abuse of 23 tramadol abusers.

\begin{tabular}{|c|c|}
\hline Variables & $N$ \\
\hline \multicolumn{2}{|l|}{ Duration of tramadol abuse (month) } \\
\hline $\bar{x} \pm s$ & $61.8 \pm 30.2$ \\
\hline Median (min-max) & $63.0(5-112)$ \\
\hline \multicolumn{2}{|l|}{ Age of first tramadol use (year) } \\
\hline $\bar{x} \pm s$ & $18.3 \pm 4.0$ \\
\hline Median (min-max) & $18.0(13-28)$ \\
\hline \multicolumn{2}{|l|}{ Purpose of first tramadol use } \\
\hline $\begin{array}{l}\text { Instigating by friends or other } \\
\text { acquaintances }\end{array}$ & 14 \\
\hline Curiosity or simulating & 9 \\
\hline Medical purpose & 2 \\
\hline $\begin{array}{l}\text { Experienced the spiritual effects of drugs } \\
\text { (euphoria) }\end{array}$ & 2 \\
\hline Relieving negative emotions & 2 \\
\hline Influence of family members & 1 \\
\hline \multicolumn{2}{|l|}{ Route of administration } \\
\hline Oral & 23 \\
\hline \multicolumn{2}{|l|}{ Median dose of tramadol use (mg/per time) } \\
\hline At the outset (range) & $250.0(50-1000)$ \\
\hline Regular dose (range) & $750.0(100-5000)$ \\
\hline Maximum (range) & $2000.0(250-10000)$ \\
\hline \multicolumn{2}{|l|}{ Source of tramadol } \\
\hline Private clinic or pharmacy & 12 \\
\hline Normal pharmacy & 6 \\
\hline Black market & 6 \\
\hline Relatives or friends & 1 \\
\hline Internet & 1 \\
\hline $\begin{array}{l}\text { Voluntary detoxification for one time or } \\
\text { above }\end{array}$ & 16 \\
\hline
\end{tabular}

measures, tramadol produced a significantly greater effect than healthy placebo $(P<0.05)$. On the ARCI-MBG measures, tramadol scores were lower than heroin but higher than MA, whereas the differences were not significant $(P>$ $0.05)$. On the ARCI-PCAG measures, the scores produced by heroin were higher than those produced by tramadol $(P<$ 0.05). However, the comparison with MA did not. On the ARCI-LSD measures, the scores of tramadol did not differ from other two active drugs $(P>0.05)$.

\section{Discussion}

Using spontaneous data from the addiction unit we found that tramadol dependence fulfilling DSM-IV criteria occurs in 23 males with no known history of substance abuse. The findings indicated that tramadol appeared to produce high abuse liability within the long-term infrequent abuse by those without preexisting substance abuse and the high doses.

Tramadol is thought to have low potential for abuse, but the cases with no reported prior history of substance abuse became dependent on tramadol in our study. This may suggest that tramadol dependence in patients with no prior history of drug abuse differed from those of opiate addicts [21]. The results did not support the previous findings from experimental [22] and surveillance studies [23]. One of the possible reasons of conflicting results is the different study sample included in the study. As far as we know, this is the first study to evaluate the dependence and abuse liability of tramadol with no drug abuse history using multiple placebo as a preference. Thus we have no comparable studies to interpret and support our results. However, a wide abuse of tramadol does not largely appear to occur in other countries, and some controversy is driven by uncertainty in subpopulation vulnerable to tramadol dependence. Chinese tramadol abuse seems to be a growing problem and a unique phenomenon. We claimed that it may be due to a complex and polymorphic pharmacokinetic or pharmacodynamics characteristics or patient characteristics. The work of further exploring the effect of those factors in tramadol dependence is the guarantee for scientific and reasonable tramadol use. Another explanation may be a discrepancy between doses used in controlled studies and doses used in real life. The actual problem is that most tramadol abusers have a long duration of abusing drugs rather than short period in controlled study. Doses of tramadol in our sample extremely exceed the doses prescribed by the physician. Results from our study suggest that high dosage with a long abused time probably increase the ability of tramadol to induce dependence.

In this study, we examined the subjective effects of tramadol compared with multiple controls using ARCI-CV. There was good concordance between tramadol in general population in our study and the findings for this drug in opiate addicts [21] regarding its subjective effects. In particular, in the results of this study, particularly those for the comparison between tramadol and heroin, no statistically significant results were found on measures of MBG scale, which suggests that the euphoria effects of tramadol is somewhat qualitatively equal to heroin. As an example of the severity of tramadol dependence, this study implies that dependence of tramadol has led to admission to drug dependence clinic of hospital for detoxification or dose reduction. The proportion of subjects having at least one time or above detoxification treatment is $69.6 \%(16 / 23)$. Otherwise, neither animals experiment nor volunteer self-administration test has been associated with reports of actual abuse. Tramadol which did not produce a significant dependence effect and no abuse potential in the laboratory did not translate into actual abuse in the marketplace. Actual abuse of drugs is influenced by a number of factors that are not assessed in human laboratory studies of abuse potential, including the availability of other abused drugs, the cost or difficulty of obtaining the drug, expectations regarding social roles, and the potential consequences of abuse $[24,25]$. In our study, it is possible that the doses much higher than the therapeutic range have contributed to its actual abuse. However, the association between higher dose and tramadol dependence should be further explored.

There were several factors that limit the interpretation regarding the significance of the results. First, we were not able to evaluate whether a dose response existed for the 
TABLE 3: Comparison of scores of ARCI scales across groups.

\begin{tabular}{|c|c|c|c|c|}
\hline & Comparison & Mean value of difference & $95 \% \mathrm{CI}$ & $P$ \\
\hline \multirow{3}{*}{ Tramadol } & MBG versus PCAG & $2.43^{* *}$ & $0.69,4.18$ & 0.007 \\
\hline & MBG versus LSD & $2.30^{*}$ & $0.56,4.05$ & 0.010 \\
\hline & PCAG versus LSD & -0.13 & $-1.87,1.61$ & 0.882 \\
\hline \multirow{3}{*}{ MBG } & Tramadol versus heroin & -0.27 & $-1.91,1.37$ & 0.745 \\
\hline & Tramadol versus MA & 0.87 & $0.92,-0.97$ & 0.348 \\
\hline & Tramadol versus healthy & $2.58^{* *}$ & $0.88,4.28$ & 0.004 \\
\hline \multirow{3}{*}{ PCAG } & Tramadol versus heroin & $-2.18^{* *}$ & $-3.69,-0.68$ & 0.005 \\
\hline & Tramadol versus MA & -0.19 & $-1.77,1.38$ & 0.807 \\
\hline & Tramadol versus healthy & $2.02^{*}$ & $0.47,3.57$ & 0.012 \\
\hline \multirow{3}{*}{ LSD } & Tramadol versus heroin & -0.58 & $-1.79,0.62$ & 0.334 \\
\hline & Tramadol versus MA & -2.14 & $-1.47,1.04$ & 0.733 \\
\hline & Tramadol versus healthy & $1.50^{*}$ & $0.25,2.75$ & 0.020 \\
\hline
\end{tabular}

LSD test, ${ }^{*} P<0.05$, and ${ }^{* *} P<0.01$.

observed effects. Our results were limited to evaluating the higher doses of tramadol, which were higher than prescribed doses exceedingly. Second, as with any study of this type, conclusions drawn regarding the assessment of relative abuse potential for drugs depend on the patients' recollection. And the methodology is the major limitation of our study. We would improve and amend it in the following research. Third, the population tested in this study would not necessarily be expected to be representative of all tramadol abusers. Fourth, the sample is small. The reasons and its importance have been discussed above.

In conclusion, these results add further support to tramadol dependence data indicating that tramadol has a high risk of producing dependence potential. A history of tramadol abuse with a long period and/or high doses may be one of important risk factors for those with no prior drug abuse history. To further strengthen the surveillance and administration of tramadol would be necessary.

\section{Conflict of Interests}

All authors declare that they have no conflict of interests.

\section{Acknowledgments}

The authors would especially like to thank the staff at Medical Hospital of Guangzhou City of Guangdong province and detoxification center in Beijing. The survey would not have been possible without their hard work. Also we gratefully thank the participants for agreeing to take part in the investigation. This study was funded by a Grant from the Major State Basic Research Project (973 Program, Grant no. 2009CB522007) and the National "Twelfth Five-Year" Plan for Science and Technology Support Program (Grant no. 2012BAI01B07) of China.

\section{References}

[1] C. Gillen, M. Haurand, D. J. Kobelt, and S. Wnendt, "Affinity, potency and efficacy of tramadol and its metabolites at the cloned human $\mu$-opioid receptor," Naunyn-Schmiedeberg's Archives of Pharmacology, vol. 362, no. 2, pp. 116-121, 2000.

[2] L. Radbruch, S. Grond, and K. A. Lehmann, "A risk-benefit assessment of tramadol in the management of pain," Drug Safety, vol. 15, no. 1, pp. 8-29, 1996.

[3] WHO Expert Committee on Drug Dependence, Twenty-Eighth Report, WHO, Geneva, Switzerland, 1992.

[4] WHO Expert Committee on Drug Dependence, Thirty-Second Report, WHO, Geneva, Switzerland, 2000.

[5] WHO Expert Committee on Drug Dependence, Thirty-Third Report, WHO, Geneva, Switzerland, 2002.

[6] WHO Expert Committee on Drug Dependence, Thirty-Fourth Report, WHO, Geneva, Switzerland, 2006.

[7] SFDA, "Narcotic drugs and psychotropic drugs directory," 7th version, 2008.

[8] SFDA, "National drug abuse monitoring center," Annual Report of National Drug Abuse, 2009.

[9] SFDA, "National drug abuse monitoring center," Annual Report of National Drug Abuse, 2010.

[10] SFDA, "National drug abuse monitoring center," Annual Report of National Drug Abuse, 2011.

[11] SFDA, "National drug abuse monitoring center," Annual Report of National Drug Abuse, 2012.

[12] Center for ADR Monitoring of Guangdong Province, "It is more terrable for analgesic addiction," 2006, http://news.sina.com.cn/c/h/2006-09-19/162511050122.shtml. 2006-09-19.

[13] H. F. Miranda and G. Pinardi, "Antinociception, tolerance, and physical dependence comparison between morphine and tramadol," Pharmacology Biochemistry and Behavior, vol. 61, no. 4, pp. 357-360, 1998.

[14] J. E. Sprague, M. Leifheit, J. Selken, M. M. Milks, D. H. Kinder, and D. E. Nichols, "In vivo microdialysis and conditioned place preference studies in rats are consistent with abuse potential of tramadol," Synapse, vol. 43, no. 2, pp. 118-121, 2002.

[15] M. Zhang, L. Jing, Q. Liu et al., "Tramadol induces conditioned place preference in rats: interactions with morphine and buprenorphine," Neuroscience Letters, vol. 520, no. 1, pp. 87-91, 2012.

[16] K. L. Preston, D. R. Jasinski, and M. Testa, "Abuse potential and pharmacological comparison of tramadol and morphine," Drug and Alcohol Dependence, vol. 27, no. 1, pp. 7-17, 1991. 
[17] M. D. Vickers, D. O’Flaherty, S. M. Szekely, M. Read, and J. Yoshizumi, "Tramadol: pain relief by an opioid without depression of respiration," Anaesthesia, vol. 47, no. 4, pp. 291296, 1992.

[18] R. J. Leo, R. Narendran, and B. Deguiseppe, "Methadone detoxification of tramadol dependence," Journal of Substance Abuse Treatment, vol. 19, no. 3, pp. 297-299, 2000.

[19] S. Shadnia, K. Soltaninejad, K. Heydari, G. Sasanian, and M. Abdollahi, "Tramadol intoxication: a review of 114 cases," Human and Experimental Toxicology, vol. 27, no. 3, pp. 201-205, 2008.

[20] J. E. Sprague, M. Leifheit, J. Selken, M. M. Milks, D. H. Kinder, and D. E. Nichols, "In vivo microdialysis and conditioned place preference studies in rats are consistent with abuse potential of tramadol," Synapse, vol. 43, no. 2, pp. 118-121, 2002.

[21] Z.-M. Liu, W.-H. Zhou, Z. Lian et al., "Drug dependence and abuse potential of tramadol," Acta Pharmacologica Sinica, vol. 20, no. 1, pp. 52-54, 1999.

[22] E. C. O'Connor and A. N. Mead, "Tramadol acts as a weak reinforcer in the rat self-administration model, consistent with its low abuse liability in humans," Pharmacology Biochemistry and Behavior, vol. 96, no. 3, pp. 279-286, 2010.

[23] T. J. Cicero, E. H. Adams, A. Geller et al., "A postmarketing surveillance program to monitor ultram (tramadol hydrochloride) abuse in the united states," Drug and Alcohol Dependence, vol. 57, no. 1, pp. 7-22, 1999.

[24] B. Dew, K. Elifson, and M. Dozier, "Social and environmental factors and their influence on drug use vulnerability and resiliency in rural populations," Journal of Rural Health, vol. 23, no. 1, supplement, pp. 16-21, 2007.

[25] A. Fletcher, C. Bonell, and J. Hargreaves, "School effects on young people's drug use: a systematic review of intervention and observational studies," Journal of Adolescent Health, vol. 42, no. 3, pp. 209-220, 2008. 


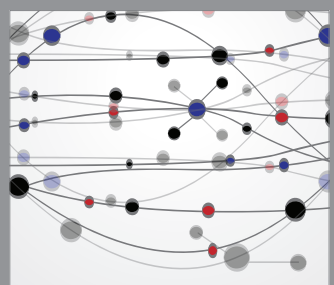

The Scientific World Journal
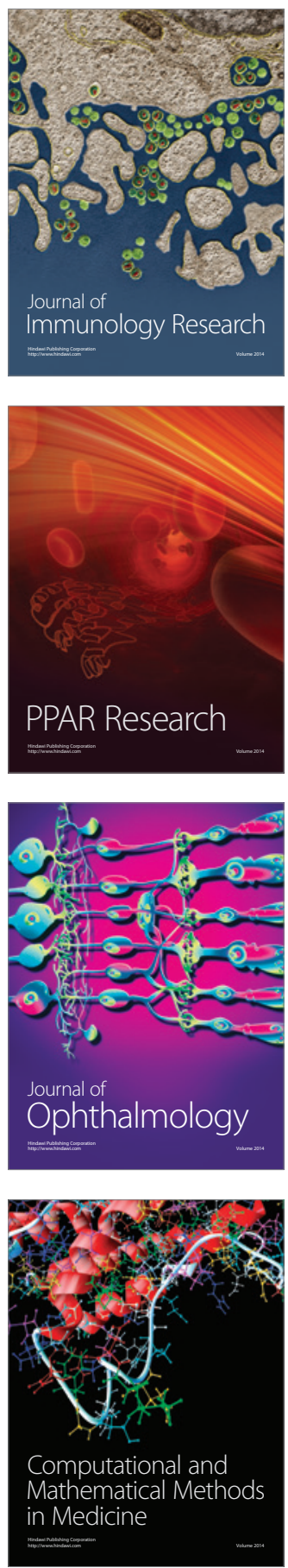

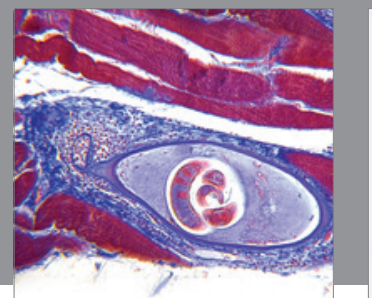

Gastroenterology

Research and Practice
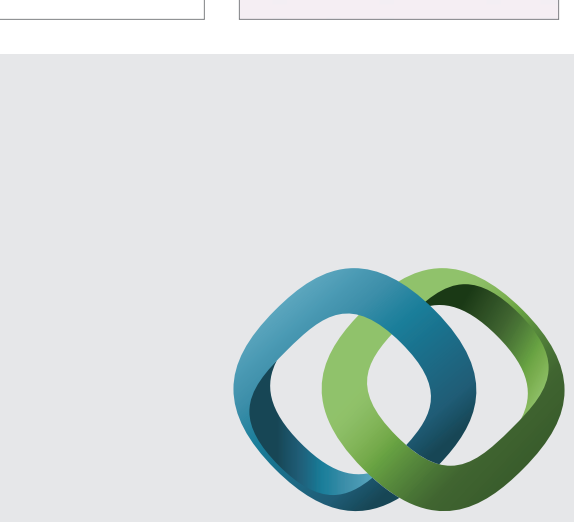

\section{Hindawi}

Submit your manuscripts at

http://www.hindawi.com
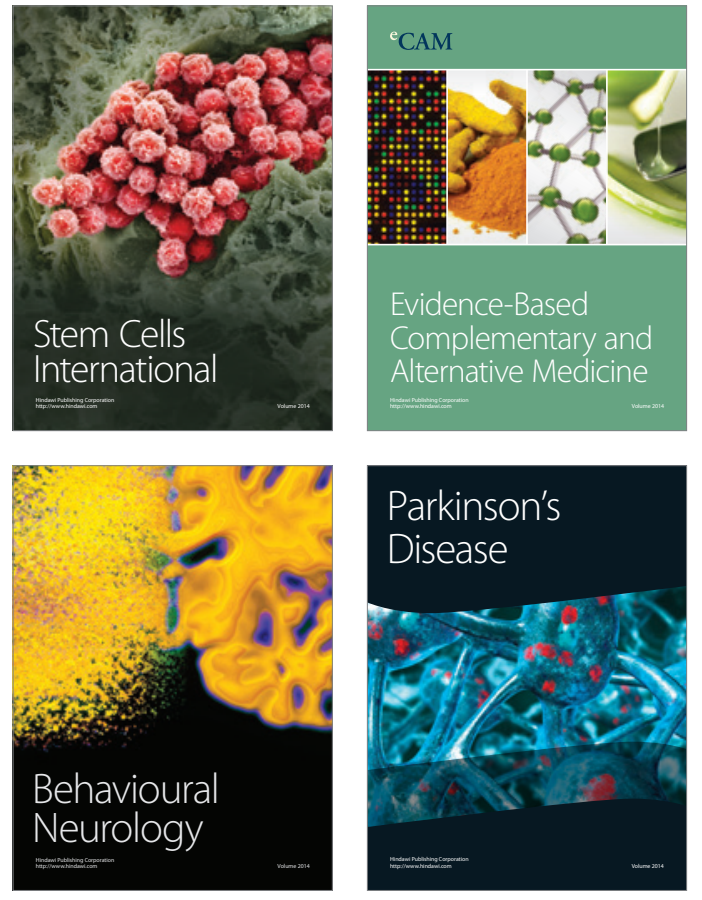
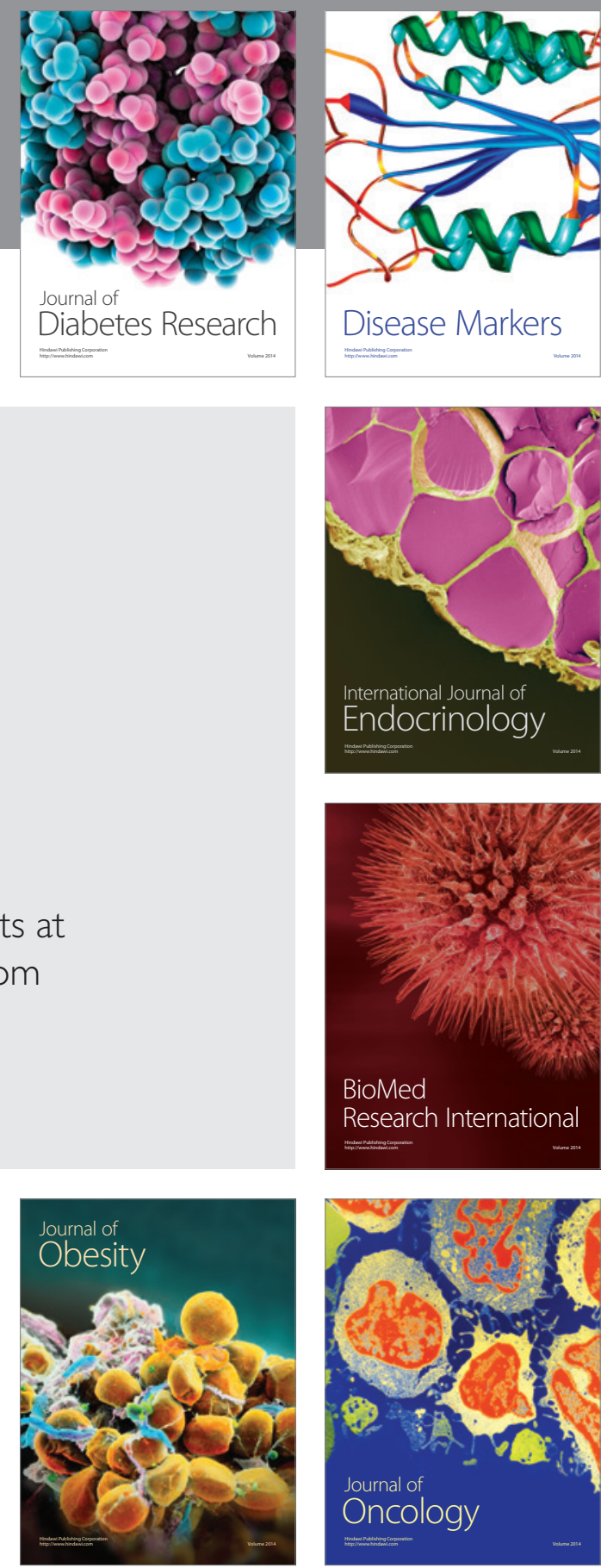

Disease Markers
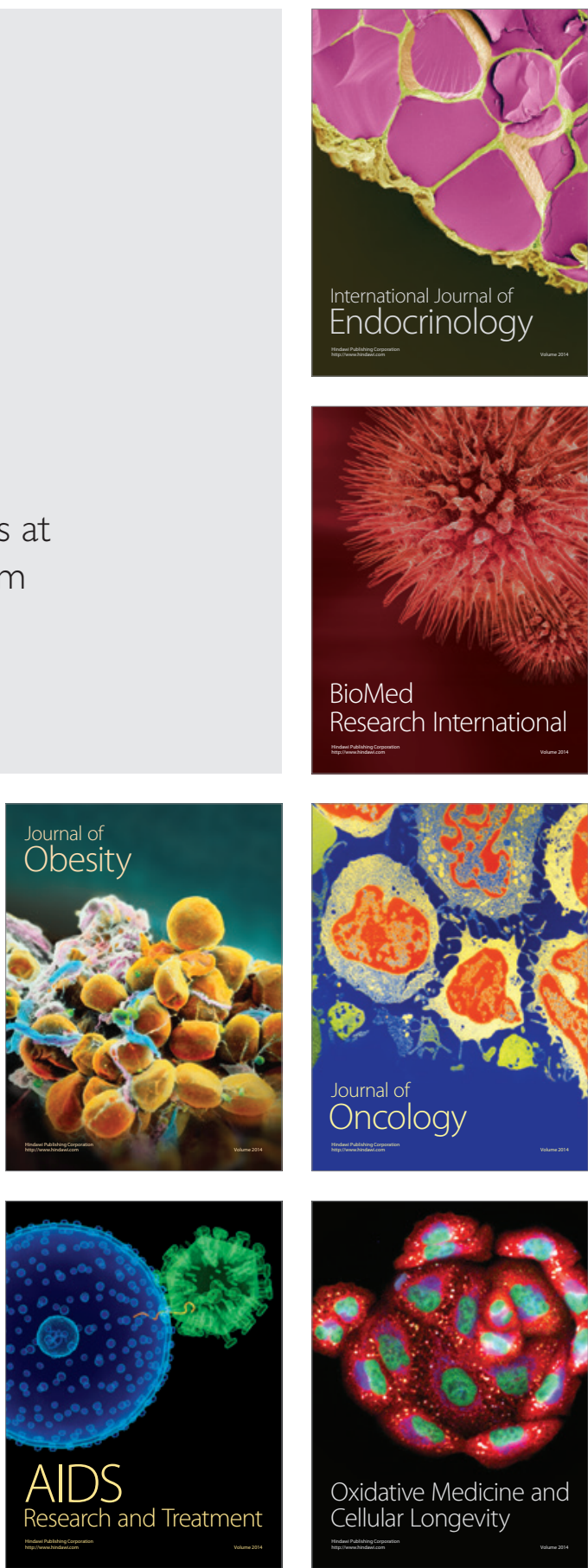